PROCEEDINGS OF THE

AMERICAN MATHEMATICAL SOCIETY

Volume 139, Number 5, May 2011, Pages 1763-1776

S 0002-9939(2010)10666-4

Article electronically published on October 29, 2010

\title{
AN INFINITY LAPLACE EQUATION WITH GRADIENT TERM AND MIXED BOUNDARY CONDITIONS
}

\author{
SCOTT N. ARMSTRONG, CHARLES K. SMART, AND STEPHANIE J. SOMERSILLE
}

(Communicated by Matthew J. Gursky)

\begin{abstract}
We obtain existence, uniqueness, and stability results for the modified 1-homogeneous infinity Laplace equation

$$
-\Delta_{\infty} u-\beta|D u|=f,
$$

subject to Dirichlet or mixed Dirichlet-Neumann boundary conditions. Our arguments rely on comparing solutions of the PDE to subsolutions and supersolutions of a certain finite difference approximation.
\end{abstract}

\section{INTRODUCTION}

Recently, the first two authors [1, 2] observed that solutions of the 1-homogeneous infinity Laplace equation

$$
-\Delta_{\infty} u:=|D u(x)|^{-2}\left\langle D^{2} u(x) D u(x), D u(x)\right\rangle=f(x)
$$

can be perturbed by $O(\varepsilon)$ to produce subsolutions and supersolutions of an $\varepsilon$ step finite difference equation. This observation was repeatedly employed in [2] to simplify and generalize many aspects of the theory for the PDE (1.1).

In this article, we extend this idea to the mixed Dirichlet-Neumann boundaryvalue problem

$$
\begin{cases}-\Delta_{\infty} u-\beta|D u|=f & \text { in } \Omega, \\ D_{\nu} u=0 & \text { on } \Gamma_{N}, \\ u=g & \text { on } \Gamma_{D},\end{cases}
$$

where $\Omega \subseteq \mathbb{R}^{n}$ is a smooth bounded open set, $\Gamma_{D} \cup \Gamma_{N}=\partial \Omega$ is a partition of $\partial \Omega$ with $\Gamma_{D}$ nonempty and closed, $\nu$ is the outer unit normal vector to $\partial \Omega$, and $\beta \in \mathbb{R}$. In the case $\Gamma_{N} \neq \emptyset$, we also require $\Omega$ to be convex. Our main result, Theorem 2.2. extends 2, Proposition 5.3] to the PDE in (1.2). It states that a subsolution of (1.2) becomes a subsolution of a certain finite difference equation after we max over $\varepsilon$-balls. This result allows us to obtain new existence, uniqueness, and stability results for the boundary-value problem (1.2) via arguments which are self-contained and elementary.

Peres, Schramm, Sheffield, and Wilson [10] showed that the infinity Laplace equation describes the continuum limit of the value functions of a two-player, randomturn game called $\varepsilon$-step tug-of-war. A biased version of $\varepsilon$-step tug-of-war, in which

Received by the editors November 1, 2009 and, in revised form, May 23, 2010.

2010 Mathematics Subject Classification. Primary 35J70, 35J75, 91A15.

Key words and phrases. Infinity Laplace equation, comparison principle.

(C)2010 American Mathematical Society 1763

Reverts to public domain 28 years from publication 
the randomness favors one of the players, gives rise to the PDE in (1.2) with $\beta \neq 0$, as shown by Peres, Pete, and the third author [9] in the case $f \equiv 0$ and $\Gamma_{N}=\emptyset$. Probabilistic arguments have also been used by Charro, García Azorero, and Rossi [4] to obtain existence of solutions to the infinity Laplace equation with mixed boundary conditions. While we do not use any probabilistic arguments in this paper, the finite difference equation we introduce below does correspond to a certain biased $\varepsilon$-step tug-of-war game. Thus, many of our techniques and arguments have probabilistic analogues. However, the finite difference equation we consider here is designed with analytic consequences in mind (particularly Theorem 2.2).

In each of the articles mentioned in the previous paragraph, solutions of the infinity Laplace equation are obtained by computing the limit as $\varepsilon \rightarrow 0$ of the value functions of an $\varepsilon$-step tug-of-war game. In contrast, in this paper we first establish an analogue of comparisons with cones, which allows us to apply the Perron process to establish the existence of maximal and minimal solutions of our boundary-value problem. This approach to existence is a simplification of a method employed recently by Lu and Wang $[8]$ in the case $\beta=0, \Gamma_{N}=\emptyset$.

We reduce our uniqueness and stability theorems to corresponding results for the finite difference equation by applying the perturbation theorem (Theorem 2.2). The proofs of the latter are straightforward since the finite difference equation is simpler to analyze.

While we make no use of probabilistic methods in the paper, let us briefly recall from 9 the two-player, random-turn, biased tug-of-war game which gives rise to our finite difference equation (2.3), below. Given are a step size $\varepsilon>0$; a parameter $\beta \in \mathbb{R}$; a domain $\Omega$ with boundary partitioned into a nonempty, closed Dirichlet piece $\Gamma_{D}$, and a Neumann piece $\Gamma_{N}$; a running payoff function $f \in C\left(\Omega \cup \Gamma_{N}\right) \cap$ $L^{\infty}\left(\Omega \cup \Gamma_{N}\right)$; a final payoff function $g \in C\left(\Gamma_{D}\right)$; and a starting position $x_{0} \in \bar{\Omega}$. At the $k$ th stage of the game, a biased coin is tossed for which the odds of Player I winning are $a_{\varepsilon}^{+}(\beta)$ to $a_{\varepsilon}^{-}(\beta)$, where $a_{\varepsilon}^{ \pm}(\beta)$ are given by (2.4). That is, if $\beta \neq 0$, then

$$
\text { Probability of Player I winning the coin toss }=\frac{a_{\varepsilon}^{+}(\beta)}{a_{\varepsilon}^{+}(\beta)+a_{\varepsilon}^{-}(\beta)}=\frac{\exp (\varepsilon \beta)}{1+\exp (\varepsilon \beta)} \text {, }
$$

while the probability of Player II winning the toss is $\exp (-\varepsilon \beta) /(1+\exp (-\varepsilon \beta))$; if $\beta=0$, then the coin is fair. The winner of the toss then selects a point $x_{k} \in$ $\bar{B}\left(x_{k-1}, \varepsilon\right) \cap \bar{\Omega}$, and the token is moved from $x_{k-1}$ to $x_{k}$. This completes the $k$ th stage of the game. Play continues until $x_{m} \in \Gamma_{D}$, at which point the game is ended and Player II pays Player I the amount

$$
\text { Payoff }=g\left(x_{m}\right)+\frac{\varepsilon}{\beta}\left(\frac{\exp (\varepsilon \beta)-1}{\exp (\varepsilon \beta)+1}\right) \sum_{k=1}^{m} f\left(x_{k-1}\right) .
$$

Observe that the term multiplying the running payoff sum is approximately $\varepsilon^{2} / 2$ for small $\varepsilon$ or $|\beta|$, and we replace it by $\varepsilon^{2} / 2$ in the case that $\beta=0$. If the token never reaches $\Gamma_{D}$ and the game thus fails to terminate, each of the players must pay a fine of $+\infty$. The value function $V_{I}\left(x_{0}\right)$ for Player I is, roughly, the minimum that Player I can expect to win by playing optimally, while the value function $V_{I I}\left(x_{0}\right)$ is the maximum that Player II can expect to be required to pay, by playing optimally (these are defined in a rigorous way in [9]).

It follows from the dynamic programming principle that both value functions $V_{I}$ and $V_{I I}$, as functions of the starting position $x_{0}$, satisfy the finite difference 
equation (2.3) with $h=f$. If $x_{0} \in \Gamma_{D}$, then the game is over before the first stage, and thus $V_{I}\left(x_{0}\right)=V_{I I}\left(x_{0}\right)=g\left(x_{0}\right)$. We expect that in the limit $\varepsilon \rightarrow 0$, the value functions will converge to solutions of the problem (1.2). In the special case $f=0$ and $\Gamma_{N}=\emptyset$, this was proved in 9 . We do not prove this result here, but note that it can be deduced from Theorem 2.2 in the same way that Theorem 2.11 of 2 was deduced from Proposition 5.3 in that paper.

We refer to Barron, Evans, and Jensen [3] for further random-turn games which give rise to PDEs related to the infinity Laplacian.

In the next section, we review the notion of viscosity solution for the mixed boundary-value problem (1.2) and state our assumptions and main results. In Section 3 we prove the perturbation theorem. In Section 4 we prove existence of solutions to (1.2) and apply the perturbation theorem to obtain uniqueness and stability results for our boundary-value problem.

\section{Statement of MAin RESUlts}

We take $\Omega \subseteq \mathbb{R}^{n}$ to be a bounded open connected domain with a $C^{1}$ boundary $\partial \Omega$. We partition the boundary $\partial \Omega$ into subsets $\Gamma_{N}=\partial \Omega \cap A$ and $\Gamma_{D}=\partial \Omega \backslash A \neq \emptyset$, for some open subset $A \subseteq \mathbb{R}^{n}$. In the case $\Gamma_{N} \neq \emptyset$, we require the domain $\Omega$ to be convex. We denote the outward pointing unit normal vector to $\partial \Omega$ by $\nu$.

Let us recall the notion of viscosity solution of (1.2). Define for $\varphi \in C^{2}\left(\mathbb{R}^{n}\right)$ the operators

$$
\Delta_{\infty}^{+} \varphi(x):= \begin{cases}|D \varphi(x)|^{-2}\left\langle D^{2} \varphi(x) D \varphi(x), D \varphi(x)\right\rangle & \text { if } D \varphi(x) \neq 0, \\ \max \left\{\left\langle D^{2} \varphi(x) v, v\right\rangle:|v|=1\right\} & \text { if } D \varphi(x)=0,\end{cases}
$$

and $\Delta_{\infty}^{-} \varphi(x):=-\Delta_{\infty}^{+}(-\varphi)(x)$. We denote the set of real-valued upper semicontinuous functions on a set $V \subseteq \mathbb{R}^{n}$ by $\operatorname{USC}(V)$ and the set of real-valued lower semicontinuous functions by $\operatorname{LSC}(V)$. The set of Lipschitz functions on $V$ is denoted by $\operatorname{Lip}(V)$, and the $\operatorname{Lipschitz}$ constant of $u \in \operatorname{Lip}(V)$ is denoted by $\operatorname{Lip}(u, V)$. The oscillation of a function $u: V \rightarrow \mathbb{R}$ is denoted by osc $u:=\sup _{V} u-\inf _{V} u$.

Definition 2.1. Given $h \in \operatorname{USC}\left(\Omega \cup \Gamma_{N}\right)$, we say that $u \in \operatorname{USC}(\bar{\Omega})$ is a viscosity subsolution of the system

$$
\begin{cases}-\Delta_{\infty} u-\beta|D u|=h & \text { in } \Omega \\ D_{\nu} u=0 & \text { on } \Gamma_{N}\end{cases}
$$

if for every $\varphi \in C^{2}(\bar{\Omega})$ and $x_{0} \in \Omega \cup \Gamma_{N}$ such that the map $x \mapsto(u-\varphi)(x)$ has a local maximum at $x_{0}$, we have

$$
-\Delta_{\infty}^{+} \varphi\left(x_{0}\right)-\beta\left|D \varphi\left(x_{0}\right)\right| \leq h\left(x_{0}\right)
$$

or

$$
x_{0} \in \Gamma_{N} \quad \text { and } \quad D \varphi\left(x_{0}\right) \cdot \nu\left(x_{0}\right) \leq 0 .
$$

Similarly, if $h \in \operatorname{LSC}\left(\Omega \cup \Gamma_{N}\right)$, then $v \in \operatorname{LSC}(\bar{\Omega})$ is a viscosity supersolution of (2.1) if $-v$ is a subsolution of (2.1) with $h$ replaced by $-h$. If $h \in C\left(\Omega \cup \Gamma_{N}\right)$, then $u \in C(\bar{\Omega})$ is a viscosity solution of (2.1) if it is both a viscosity subsolution and a viscosity supersolution of (2.1).

We remark that by altering Definition 2.1 by requiring all local extrema to be strict, we obtain an equivalent definition of viscosity subsolution and viscosity supersolution. We emphasize that all differential inequalities in this paper involving 
functions not known to be smooth are to be understood in the viscosity sense. In particular, if we say that $u$ is a solution of the system of differential inequalities

$$
\begin{cases}-\Delta_{\infty} u-\beta|D u| \leq h & \text { in } \Omega, \\ D_{\nu} u \leq 0 & \text { on } \Gamma_{N},\end{cases}
$$

this is taken to mean that $u$ is a viscosity subsolution of (2.1).

For each $\varepsilon>0$, we define $\Omega_{\varepsilon}$ to be the set of points in $\bar{\Omega}$ which are farther than $\varepsilon$ from the Dirichlet boundary $\Gamma_{D}$, that is,

$$
\Omega_{\varepsilon}:=\left\{x \in \bar{\Omega}: \operatorname{dist}\left(x, \Gamma_{D}\right)>\varepsilon\right\} .
$$

If $h,-\tilde{h} \in \operatorname{USC}\left(\Omega \cup \Gamma_{N}\right)$ and $\varepsilon>0$, we denote

$$
h^{\varepsilon}(x):=\max _{\bar{B}(x, \varepsilon) \cap \bar{\Omega}} h \text { and } \tilde{h}_{\varepsilon}(x):=\min _{\bar{B}(x, \varepsilon) \cap \bar{\Omega}} \tilde{h}, \quad \text { for } x \in \Omega_{\varepsilon} .
$$

For $u \in C\left(\Omega \cup \Gamma_{N}\right)$, we define the quantities

$$
S_{\varepsilon}^{+} u(x):=\frac{1}{\varepsilon}\left(u^{\varepsilon}(x)-u(x)\right) \quad \text { and } \quad S_{\varepsilon}^{-} u(x):=\frac{1}{\varepsilon}\left(u(x)-u_{\varepsilon}(x)\right) .
$$

Our finite difference approximation to (2.1) is the scheme

$$
a_{\varepsilon}^{-}(\beta) S_{\varepsilon}^{-} u(x)-a_{\varepsilon}^{+}(\beta) S_{\varepsilon}^{+} u(x)=h(x),
$$

where the coefficients $a_{\varepsilon}^{ \pm}(\beta)$ are given by

$$
a_{\varepsilon}^{+}(\beta):=\frac{\beta}{1-\exp (-\varepsilon \beta)} \quad \text { and } \quad a_{\varepsilon}^{-}(\beta):=\frac{\beta}{\exp (\varepsilon \beta)-1}, \quad \beta \in \mathbb{R} \backslash\{0\},
$$

and $a_{\varepsilon}^{+}(0):=a_{\varepsilon}^{-}(0):=1 / \varepsilon$. Observe that the maps $\beta \mapsto a_{\varepsilon}^{ \pm}(\beta)$ are continuous and that we have $a_{\varepsilon}^{ \pm}(-\beta)=a_{\varepsilon}^{\mp}(\beta)$.

Our main result is the following perturbation theorem.

Theorem 2.2. Suppose that $h \in \operatorname{USC}\left(\Omega \cup \Gamma_{N}\right)$ and $u \in C\left(\Omega \cup \Gamma_{N}\right)$ is a viscosity subsolution of the system (2.1). Then for each $\varepsilon>0$,

$$
a_{\varepsilon}^{-}(\beta) S_{\varepsilon}^{-} u^{\varepsilon}(x)-a_{\varepsilon}^{+}(\beta) S_{\varepsilon}^{+} u^{\varepsilon}(x) \leq h^{2 \varepsilon}(x) \quad \text { for every } \quad x \in \Omega_{2 \varepsilon} .
$$

Theorem 2.2 asserts that small perturbations of viscosity subsolutions of (1.2) are subsolutions of the finite difference equation (2.3). We will see below that (2.3) has a simple comparison lemma (see Lemma 4.2), which we combine with Theorem 2.2 to deduce the following comparison result for (2.1).

Theorem 2.3. Assume that $h,-\tilde{h} \in \operatorname{USC}\left(\Omega \cup \Gamma_{N}\right) \cap L^{\infty}\left(\Omega \cup \Gamma_{N}\right)$ satisfy $h \leq \tilde{h}$, as well as

$$
h<\tilde{h}, \quad h \equiv 0, \quad \text { or } \quad \tilde{h}>0 .
$$

Suppose that $u \in \operatorname{USC}(\bar{\Omega})$ is a viscosity subsolution of the system

$$
\begin{cases}-\Delta_{\infty} u-\beta|D u| \leq h & \text { in } \Omega, \\ D_{\nu} u \leq 0 & \text { on } \Gamma_{N}\end{cases}
$$

and $v \in \operatorname{LSC}(\bar{\Omega})$ is a viscosity supersolution of the system

$$
\begin{cases}-\Delta_{\infty} v-\beta|D v| \geq \tilde{h} & \text { in } \Omega \\ D_{\nu} v \geq 0 & \text { on } \Gamma_{N} .\end{cases}
$$

Then

$$
\max _{\bar{\Omega}}(u-v)=\max _{\Gamma_{D}}(u-v)
$$


Notice that Theorem 2.3 implies that for $f \in C\left(\Omega \cup \Gamma_{N}\right) \cap L^{\infty}\left(\Omega \cup \Gamma_{N}\right)$ and $g \in C\left(\Gamma_{D}\right)$, the problem (1.2) has at most one solution in the cases $f \equiv 0$ and $f>0$ (and, by symmetry, $f<0$ ). While uniqueness is known to fail in general (see [10, Section 5] and Remark 4.3 below), the following theorem establishes the existence of a maximal and minimal solution of the problem (1.2) for any $f$. In particular, we deduce the unique solvability of (1.2) whenever uniqueness holds. This result extends [2, Theorem 2.14], and is obtained by a Perron argument.

Theorem 2.4. Assume that $f \in C\left(\Omega \cup \Gamma_{N}\right) \cap L^{\infty}\left(\Omega \cup \Gamma_{N}\right)$ and $g \in C\left(\Gamma_{D}\right)$. Then there exist solutions $\underline{u}, \bar{u} \in C(\bar{\Omega})$ of the boundary-value problem (1.2), with the property that $u \leq \bar{u}(u \geq \underline{u})$ if $u$ is a subsolution (supersolution) of (1.2) and $u \leq g$ $(u \geq g)$ on $\Gamma_{D}$.

With the aid of Theorem 2.2, we will prove the following stability result.

Theorem 2.5. Suppose that $\beta, \beta_{j} \in \mathbb{R}, f, f_{j} \in \mathrm{USC}\left(\Omega \cup \Gamma_{N}\right)$, and $u, u_{j} \in C\left(\Omega \cup \Gamma_{N}\right)$ such that for each $j \geq 1$, the function $u_{j}$ satisfies the system

$$
\begin{cases}-\Delta_{\infty} u_{j}-\beta_{j}\left|D u_{j}\right| \leq f_{j} & \text { in } \Omega, \\ D_{\nu} u_{j} \leq 0 & \text { on } \Gamma_{N} .\end{cases}
$$

Suppose in addition that $\beta_{j} \rightarrow \beta$, and $f_{j} \rightarrow f$ and $u_{j} \rightarrow u$ locally uniformly in $\Omega \cup \Gamma_{N}$. Then $u$ is a subsolution of the system

$$
\begin{cases}-\Delta_{\infty} u-\beta|D u| \leq f & \text { in } \Omega, \\ D_{\nu} u \leq 0 & \text { on } \Gamma_{N} .\end{cases}
$$

Combining the sup-norm and interior Lipschitz estimates (see Lemma 4.1, below) with Theorem 2.5, we immediately obtain the following result.

Corollary 2.6. Let $\beta, \beta_{j}, f, f_{j}$ be as in the hypotheses of Theorem 2.5, and suppose in addition that $\left|f_{j}\right| \leq K$ for all $j$. Assume that $g, g_{j} \in C\left(\Gamma_{D}\right)$ such that $g_{j} \rightarrow g$ uniformly, and $u_{j}$ is a solution of the problem

$$
\begin{cases}-\Delta_{\infty} u_{j}-\beta_{j}\left|D u_{j}\right|=f_{j} & \text { in } \Omega, \\ D_{\nu} u_{j}=0 & \text { on } \Gamma_{N}, \\ u_{j}=g_{j} & \text { on } \Gamma_{D} .\end{cases}
$$

Then there is a subsequence $\left\{u_{j_{k}}\right\}$ and a solution $u \in C(\bar{\Omega})$ of the problem

$$
\begin{cases}-\Delta_{\infty} u-\beta|D u|=f & \text { in } \Omega, \\ D_{\nu} u=0 & \text { on } \Gamma_{N}, \\ u=g & \text { on } \Gamma_{D},\end{cases}
$$

such that $u_{j_{k}} \rightarrow u$ uniformly on $\bar{\Omega}$.

\section{The Perturbation theorem}

We begin our proof of Theorem 2.2 by showing that viscosity solutions of (2.1) satisfy an analogue of comparisons with cones from above, a property satisfied by infinity subharmonic functions (see [5]). Here our "cones" are functions of the form $\varphi(x):=\gamma\left(\left|x-x_{0}\right|\right)$, where $\gamma$ is a solution of the ODE

$$
-\gamma^{\prime \prime}-\beta\left|\gamma^{\prime}\right|=k
$$


It is easy to see that such a function $\varphi$ is a $C^{2}$ solution of our PDE

$$
-\Delta_{\infty} \varphi-\beta|D \varphi|=k \quad \text { in } \mathbb{R}^{n} \backslash\left\{x_{0}\right\} .
$$

These functions are called exponential cones in [9], where a similar result to our Lemma 3.1 (and its converse) is proved in the case $f \equiv 0$ and $\Gamma_{N}=\emptyset$ using probability methods. Our proof is a little simpler, as we do not use explicit formulas for the cone functions or probability methods, but prefer to analyze the ODE (3.1) directly.

Lemma 3.1. Suppose that $k \in \mathbb{R}$ and that $u \in \operatorname{USC}(\bar{\Omega})$ is a subsolution of

$$
\begin{cases}-\Delta_{\infty} u-\beta|D u| \leq k & \text { in } \Omega, \\ D_{\nu} u \leq 0 & \text { on } \Gamma_{N} .\end{cases}
$$

Suppose further that $r>0$ and $\gamma \in C^{2}([0, r])$ satisfies

$$
-\gamma^{\prime \prime}-\beta\left|\gamma^{\prime}\right|=k \quad \text { and } \quad \gamma^{\prime}>0 \quad \text { in }(0, r)
$$

and define

$$
\varphi(x):=\gamma\left(\left|x-x_{0}\right|\right)
$$

for some $x_{0} \in \bar{\Omega}$. Denote $\Lambda:=\left(\partial B\left(x_{0}, r\right) \cap \bar{\Omega}\right) \cup\left(B\left(x_{0}, r\right) \cap \Gamma_{D}\right)$. Then

$$
\max _{\bar{B}\left(x_{0}, r\right) \cap \bar{\Omega}}(u-\varphi)=\max _{\Lambda \cup\left\{x_{0}\right\}}(u-\varphi) .
$$

If in addition $\gamma^{\prime}(0)=0$, then

$$
\max _{\bar{B}\left(x_{0}, r\right) \cap \bar{\Omega}}(u-\varphi)=\max _{\Lambda}(u-\varphi) .
$$

Proof. By the upper semicontinuity of $u$, we may assume that $x_{0} \in \Omega$. By the convexity of $\Omega$ in the case that $\Gamma_{N} \neq \emptyset$, we have that $\left(y-x_{0}\right) \cdot \nu>0$ for all $y \in \Gamma_{N}$.

Consider first the case that $\gamma^{\prime}(0)>0$. Arguing indirectly, suppose that the conclusion (3.3) fails so that we can find $x_{1} \in \bar{B}\left(x_{0}, r\right) \cap \bar{\Omega}$ such that

$$
(u-\varphi)\left(x_{1}\right)>\max _{\Lambda \cup\left\{x_{0}\right\}}(u-\varphi) .
$$

For $\delta, \eta \geq 0$ to be chosen below, let $\tilde{\gamma} \in C^{2}([0, r])$ be the solution of the initial-value problem

$$
-\tilde{\gamma}^{\prime \prime}-\beta\left|\tilde{\gamma}^{\prime}\right|=k+\delta, \quad \tilde{\gamma}(0)=\gamma(0), \quad \tilde{\gamma}^{\prime}(0)=\gamma^{\prime}(0)+\eta .
$$

For the choice $\eta>0$ and $\delta=0$, it is easy to check that $\tilde{\gamma}^{\prime}>\gamma^{\prime} \geq 0$ on $[0, r]$. Thus by elementary stability properties of our ODE, we can select small enough $\eta>0$ and $\delta>0$ such that $\tilde{\gamma}^{\prime}>0$ on $[0, r]$ and the function $\tilde{\varphi}(x):=\tilde{\gamma}\left(\left|x-x_{0}\right|\right)$ satisfies (3.5) with $\varphi$ replaced by $\tilde{\varphi}$.

Select a point $x_{2} \in \bar{B}\left(x_{0}, r\right) \cap \bar{\Omega}$ at which the map $x \mapsto(u-\tilde{\varphi})(x)$ attains its maximum in $\bar{B}\left(x_{0}, r\right) \cap \bar{\Omega}$, and note that $x_{2} \notin \Lambda \cup\left\{x_{0}\right\}$. Observe that $\tilde{\varphi}$ is $C^{2}$ in a neighborhood of $x_{2}$ and

$$
-\Delta_{\infty}^{-} \tilde{\varphi}\left(x_{2}\right)-\beta\left|D \tilde{\varphi}\left(x_{2}\right)\right|=k+\delta>k .
$$

Since $u$ is a viscosity subsolution of (3.2), it must be the case that $x_{2} \notin \Omega$. Thus we must have $x_{2} \in \Gamma_{N}$. Using again that $u$ is a viscosity subsolution of (3.2), we have $\nu \cdot D \varphi\left(x_{2}\right) \leq 0$. This contradicts the fact that $\tilde{\gamma}^{\prime}\left(\left|x_{2}-x_{0}\right|\right)>0$ and $\left(x_{2}-x_{0}\right) \cdot \nu>0$. We have verified (3.3) in the case $\gamma^{\prime}(0)>0$. 
In the case $\gamma^{\prime}(0)=0$ we also argue indirectly and suppose that (3.4) fails, so that there exists $x_{1} \in \bar{B}\left(x_{0}, r\right) \cap \bar{\Omega}$ such that

$$
(u-\varphi)\left(x_{1}\right)>\max _{\Lambda}(u-\varphi) .
$$

It is easy to see that $\gamma^{\prime}(0)=0$ and $\gamma^{\prime}>0$ on $(0, r)$ implies that $k<0$. For $0 \leq \delta \leq-k$ to be selected below, let $\tilde{\gamma}$ be defined by

$$
\tilde{\gamma}(t):= \begin{cases}-\frac{k+\delta}{\beta} t-\frac{k+\delta}{\beta^{2}}\left(e^{-\beta t}-1\right)+\gamma(0), & \text { if } \beta \neq 0, \\ \frac{k+\delta}{2} t^{2}+\gamma(0), & \text { if } \beta=0 .\end{cases}
$$

Then $\tilde{\gamma} \in C^{2}([0, \infty))$ satisfies $\tilde{\gamma}^{\prime}>0$ in $(0, \infty)$ and

$$
-\tilde{\gamma}^{\prime \prime}-\beta\left|\tilde{\gamma}^{\prime}\right|=k+\delta, \quad \tilde{\gamma}(0)=\gamma(0), \quad \tilde{\gamma}^{\prime}(0)=0 .
$$

For sufficiently small $0<\delta<-k$, the function $\tilde{\varphi}(x):=\tilde{\gamma}\left(\left|x-x_{0}\right|\right)$ satisfies (3.6) with $\varphi$ replaced by $\tilde{\varphi}$. Notice also that $\tilde{\varphi} \in C^{2}\left(\mathbb{R}^{n}\right)$ and

$$
-\Delta_{\infty}^{-} \tilde{\varphi}(x)-\beta|D \tilde{\varphi}(x)|=k+\delta>k, \quad x \in \mathbb{R}^{n} .
$$

Select a point $x_{2} \in \bar{B}\left(x_{0}, r\right) \cap \bar{\Omega}$ at which the map $x \mapsto(u-\tilde{\varphi})(x)$ attains its maximum in $\bar{B}\left(x_{0}, r\right) \cap \bar{\Omega}$, and note that $x_{2} \notin \Lambda$. Recalling that $x_{0} \notin \partial \Omega$, we may proceed as above to derive a contradiction.

The following lemma is a version of Theorem 2.2 for our cone functions.

Lemma 3.2. Assume that $\gamma \in C^{2}([0, r])$ satisfies

$$
-\gamma^{\prime \prime}-\beta\left|\gamma^{\prime}\right|=k \quad \text { and } \quad \gamma^{\prime}>0 \quad \text { in }(0, r) .
$$

Suppose that $\varepsilon>0$ and either $r \geq 2 \varepsilon$ or $\gamma^{\prime}(r)=0$. Then

$$
a_{\varepsilon}^{-}(\beta)\left(\gamma\left(r_{1}\right)-\gamma(0)\right)-a_{\varepsilon}^{+}(\beta)\left(\gamma\left(r_{2}\right)-\gamma\left(r_{1}\right)\right) \leq \varepsilon k,
$$

where we have set $r_{1}:=\min \{\varepsilon, r\}$ and $r_{2}:=\min \{2 \varepsilon, r\}$.

Proof. We consider only the case $\beta \neq 0$. The case $\beta=0$ is similar (and easier) and was proved in [2]. Since $\gamma^{\prime}>0$ in $(0, r)$, we see that $\gamma$ satisfies the linear equation

$$
-\gamma^{\prime \prime}-\beta \gamma^{\prime}=k \text { in }(0, r) \text {. }
$$

In particular, there are constants $c_{1}, c_{2} \in \mathbb{R}$ such that

$$
\gamma(t)=c_{1}+c_{2} e^{-\beta t}-\frac{k}{\beta} t, \quad 0 \leq t \leq r .
$$

Consider the case $r \geq 2 \varepsilon$. Then we have

$$
\gamma\left(r_{1}\right)-\gamma(0)=c_{2} e^{-\beta \varepsilon}\left(1-e^{\beta \varepsilon}\right)-\frac{k}{\beta} \varepsilon
$$

and

$$
\gamma\left(r_{2}\right)-\gamma\left(r_{1}\right)=c_{2} e^{-\beta \varepsilon}\left(e^{-\beta \varepsilon}-1\right)-\frac{k}{\beta} \varepsilon .
$$

The conclusion now follows from some algebra, making use of the identity

$$
\left(1-e^{-\beta \varepsilon}\right)^{-1}-\left(e^{\beta \varepsilon}-1\right)^{-1}=1 .
$$

Now consider the case $r<2 \varepsilon$ and $\gamma^{\prime}(r)=0$. Note that $\gamma^{\prime}(r)=0$ implies that

$$
\gamma(t)=c_{1}-\frac{k}{\beta^{2}} e^{-\beta(t-r)}-\frac{k}{\beta} t .
$$


Note also that $\gamma^{\prime}>0$ in $(0, r)$ and $\beta \neq 0$ imply that $k>0$. In particular, if we extend $\gamma$ to all of $\mathbb{R}$ by the formula above, we see that it is concave and achieves its maximum at $t=r$. Therefore, using (3.7) we obtain

$$
\gamma\left(r_{1}\right)-\gamma(0) \leq \gamma\left(r_{1}\right)-\gamma\left(r_{1}-\varepsilon\right)=c_{2} e^{-\beta r_{1}}\left(1-e^{\beta \varepsilon}\right)-\frac{k}{\beta} \varepsilon
$$

and

$$
\gamma\left(r_{2}\right)-\gamma\left(r_{1}\right) \geq \gamma\left(r_{1}+\varepsilon\right)-\gamma\left(r_{1}\right)=c_{2} e^{-\beta r_{1}}\left(e^{-\beta \varepsilon}-1\right)-\frac{k}{\beta} \varepsilon .
$$

The conclusion follows from a little algebra, as above.

Theorem 2.2 follows at once from the following lemma.

Lemma 3.3. Suppose that $k \in \mathbb{R}$ and $u \in C(\bar{\Omega})$ is a subsolution of (3.2). Then

$$
a_{\varepsilon}^{-}(\beta) S_{\varepsilon}^{-} u^{\varepsilon}(x)-a_{\varepsilon}^{+}(\beta) S_{\varepsilon}^{+} u^{\varepsilon}(x) \leq k \quad \text { for every } x \in \bar{\Omega}_{2 \varepsilon} .
$$

Proof. Suppose $u \in C(\bar{\Omega})$ satisfies (3.2) and $x \in \Omega_{2 \varepsilon}$. Let $y \in \bar{B}(x, \varepsilon) \cap \bar{\Omega}$ and $z \in \bar{B}(x, 2 \varepsilon) \cap \bar{\Omega}$ satisfy

$$
u(y)=u^{\varepsilon}(x) \text { and } \quad u(z)=u^{2 \varepsilon}(x),
$$

and observe that

$$
\varepsilon S_{\varepsilon}^{-} u^{\varepsilon}(x) \leq u(y)-u(x) \text { and } \varepsilon S_{\varepsilon}^{+} u^{\varepsilon}(x)=u(z)-u(y) .
$$

Select $\delta>0$, and let $\gamma \in C^{2}([0, r])$ be the solution of the initial-value problem

$$
-\gamma^{\prime \prime}-\beta\left|\gamma^{\prime}\right|=k, \quad \gamma(0)=u(x), \quad \gamma^{\prime}(0)=\eta,
$$

where $\eta$ is minimized subject to the constraints $\eta \geq 0$ and $\max _{[0,2 \varepsilon]} \gamma \geq u(z)+\delta$. Set $r:=\inf \{s>0: \gamma(s) \geq u(z)+\delta\}$, and notice that $\gamma(r)=u(z)+\delta>u(x)=\gamma(0)$, and thus $0<r \leq 2 \varepsilon$.

We claim that $\eta>0$. Suppose on the contrary that $\eta=0$. Since $\gamma$ is not constant, it is easy to see that its derivative $\gamma^{\prime}(t)$ can only vanish at a single point $t \in \mathbb{R}$. Since $\gamma^{\prime}(0)=0$ and $\gamma(r)>\gamma(0)$, we deduce that $\gamma^{\prime}>0$ on $(0, \infty)$. Define $\varphi(w):=\gamma(|w-x|)-\delta$, and notice that $\varphi \geq u(z) \geq u$ on $\partial B(x, 2 \varepsilon) \cap \bar{\Omega}$. From Lemma 3.1 we deduce that $\varphi \geq u$ in $B(x, 2 \varepsilon) \cap \bar{\Omega}$, which is not possible since $\varphi(x)=u(x)-\delta<u(x)$. Thus $\eta>0$. It follows that $\max _{[0,2 \varepsilon]} \gamma=u(z)+\delta$.

Next we show that

$$
\gamma^{\prime}>0 \quad \text { in }(0, r), \quad \text { as well as } \quad r=2 \varepsilon \text { or } \gamma^{\prime}(r)=0 .
$$

As mentioned above, since $\gamma$ is not constant, its derivative $\gamma^{\prime}(t)$ can only vanish at a single point $t \in \mathbb{R}$. Moreover, at this point, $\gamma^{\prime}$ must change sign. Owing to the initial condition $\gamma^{\prime}(0)=\eta>0$, we see that if $\gamma^{\prime}(t)=0$ for some $t>0$, then $\gamma$ achieves its maximum at $t$. Since $\max _{[0,2 \varepsilon]} \gamma=u(z)+\delta=\gamma(r)$, the claim (3.10) follows.

Define $\tilde{\varphi}(w):=\gamma(|w-x|)$. Since $\tilde{\varphi} \geq u$ on $\{x\} \cup \partial B(x, r) \cap \bar{\Omega}$, Lemma 3.1 implies that $\tilde{\varphi} \geq u$ on $\bar{B}(x, r) \cap \bar{\Omega}$. In particular, $u(y) \leq \gamma(\min \{r, \varepsilon\})$. Since $u(z)+\delta=\gamma(r)=\gamma(\min \{r, 2 \varepsilon\})$, the conclusion for $x \in \Omega_{2 \varepsilon}$ follows from Lemma 3.2 and (3.9) once we send $\delta \rightarrow 0$. For $x \in \bar{\Omega}_{2 \varepsilon} \backslash \Omega_{2 \varepsilon}$, we obtain the conclusion by the continuity of $u$. 
Proof of Theorem 2.2. Our hypotheses imply that at each point $x_{0} \in \Omega_{2 \varepsilon}$,

$$
\begin{cases}-\Delta_{\infty} u-\beta|D u| \leq h^{2 \varepsilon}\left(x_{0}\right) & \text { in } \Omega \cap B\left(x_{0}, 2 \varepsilon\right), \\ D_{\nu} u \leq 0 & \text { on } \Gamma_{N} \cap \bar{B}\left(x_{0}, 2 \varepsilon\right) .\end{cases}
$$

Lemma 3.3 now implies that (2.5) holds for $x=x_{0}$, since $x_{0}$ belongs to the closure of the set $\left(\Omega \cap B\left(x_{0}, 2 \varepsilon\right)\right)_{2 \varepsilon}$, which is defined in the obvious way, i.e., as in (2.2) with $\Omega$ and $\Gamma_{D}$ replaced by $\Omega \cap B\left(x_{0}, 2 \varepsilon\right)$ and $\Gamma_{D} \cap \bar{B}\left(x_{0}, 2 \varepsilon\right)$, respectively.

\section{Existence, Uniqueness, AND STABiLity}

In this section we prove the rest of our main results, Theorems 2.3, 2.4, and 2.5, First, we need a sup-norm and Lipschitz estimate for subsolutions of (2.1), which we obtain with the help of Lemma 3.1.

Lemma 4.1. Suppose that $k \geq 0$ and that $u \in \operatorname{USC}(\bar{\Omega})$ is a subsolution of

$$
\begin{cases}-\Delta_{\infty} u-\beta|D u| \leq k & \text { in } \Omega \\ D_{\nu} u \leq 0 & \text { on } \Gamma_{N}\end{cases}
$$

Then

$$
\max _{\bar{\Omega}} u \leq \max _{\Gamma_{D}} u+C_{1}
$$

where the constant $C_{1}>0$ depends only on $k, \beta$, and $\operatorname{diam}(\Omega)$. If in addition $u$ is bounded below, then $u \in \operatorname{Lip}\left(\Omega_{\delta}\right)$ for each $\delta>0$, and we have the estimate

$$
|u(x)-u(y)| \leq C_{2}|x-y| \quad \text { for every } x, y \in \Omega_{\delta},
$$

where the constant $C_{2}$ depends only on $\operatorname{osc} u, \delta, k, \beta$, and $\operatorname{diam}(\Omega)$.

Proof. Denote $d:=\operatorname{diam}(\Omega)$ and define a function $\gamma=\gamma(t)$ by

$$
\gamma(t):= \begin{cases}-\frac{1}{\beta} k t-\frac{1}{\beta^{2}} k e^{\beta d}\left(e^{-\beta t}-1\right) & \text { if } \beta \neq 0, \\ -\frac{1}{2} k t^{2}+d k t & \text { if } \beta=0 .\end{cases}
$$

Observe that $\gamma$ satisfies $\gamma(0)=0$,

$$
-\gamma^{\prime \prime}-\beta\left|\gamma^{\prime}\right|=k \quad \text { in }(0, d), \quad \text { and } \quad \gamma^{\prime}>0 \quad \text { in }(0, d) .
$$

Select $x_{0} \in \Gamma_{D}$, and set

$$
\varphi(x):=\max _{\Gamma_{D}} u+\gamma\left(\left|x-x_{0}\right|\right) .
$$

By Lemma 3.1, $u \leq \varphi$ on $\bar{\Omega}$. In particular,

$$
u \leq \max _{\bar{\Omega}} \varphi \leq \max _{\Gamma_{D}} u+\gamma(d)
$$

Thus we have the estimate (4.1) for $C_{1}:=\gamma(d)$.

If $u$ is bounded below and $\delta>0$, then for any $x \in \Omega_{\delta}$ and $y \in B(x, \delta) \cap \bar{\Omega}$,

$$
u(y) \leq \varphi^{x}(y):=u(x)+\max \{1, \operatorname{osc} u / \gamma(\delta)\} \gamma(|y-x|) .
$$

This implies that $\operatorname{Lip}\left(u, \Omega_{\delta}\right) \leq \max \{1, \operatorname{osc} u / \gamma(\delta)\} \gamma^{\prime}(0)=: C_{2}$.

We now establish a comparison result for solutions of our finite difference equation. As in [1, 2], our simple argument follows that of Le Gruyer and Archer [7] (see also Le Gruyer [6]). We include a proof for completeness. 
Lemma 4.2. Suppose $u, v \in C(\bar{\Omega})$ and $f, \tilde{f}: \Omega_{\varepsilon} \rightarrow \mathbb{R}$ satisfy

$$
a_{\varepsilon}^{-}(\beta) S_{\varepsilon}^{-} u-a_{\varepsilon}^{+}(\beta) S_{\varepsilon}^{+} u \leq f \leq \tilde{f} \leq a_{\varepsilon}^{-}(\beta) S_{\varepsilon}^{-} v-a_{\varepsilon}^{+}(\beta) S_{\varepsilon}^{+} v \quad \text { in } \Omega_{\varepsilon} .
$$

Suppose in addition that (i) $f<\tilde{f}$, (ii) $f \leq 0$, or (iii) $\tilde{f} \geq 0$. Then

$$
\max _{\bar{\Omega}}(u-v)=\max _{\bar{\Omega} \backslash \Omega_{\varepsilon}}(u-v) .
$$

Proof. Arguing indirectly, let us suppose that the hypothesis holds but the conclusion fails. Define

$$
E:=\left\{x \in \bar{\Omega}:(u-v)(x)=\max _{\bar{\Omega}}(u-v)\right\},
$$

and observe that $E$ is nonempty, closed, and contained in $\Omega_{\varepsilon}$. Select $x_{0} \in E$. Since the map $x \mapsto(u-v)(x)$ attains its maximum at $x_{0}$, we have

$$
S_{\varepsilon}^{-} u\left(x_{0}\right) \geq S_{\varepsilon}^{-} v\left(x_{0}\right) \quad \text { and } \quad S_{\varepsilon}^{+} u\left(x_{0}\right) \leq S_{\varepsilon}^{+} v\left(x_{0}\right) .
$$

Since $f\left(x_{0}\right) \leq \tilde{f}\left(x_{0}\right)$, and $a_{\varepsilon}^{ \pm}(\beta)>0$, the inequalities in (4.3) must be equalities at $x_{0}$, and thus $f\left(x_{0}\right)=\tilde{f}\left(x_{0}\right)$,

$$
S_{\varepsilon}^{-} u\left(x_{0}\right)=S_{\varepsilon}^{-} v\left(x_{0}\right) \text { and } S_{\varepsilon}^{+} u\left(x_{0}\right)=S_{\varepsilon}^{+} v\left(x_{0}\right) .
$$

In particular, in the case that (i) holds we obtain a contradiction.

We have left to consider the cases (ii) and (iii). By symmetry, we need only consider (ii). Define

$$
F:=\left\{x \in E: u(x)=\max _{E} u\right\}
$$

and notice that $F$ is nonempty, closed, and $F \subseteq E \subseteq \Omega_{\varepsilon}$.

Suppose there is an $x_{0} \in F$ and an $x_{1} \in \bar{B}\left(x_{0}, \varepsilon\right)$ such that

$$
u\left(x_{1}\right)-u\left(x_{0}\right)=\varepsilon S_{\varepsilon}^{+} u\left(x_{0}\right)>0 .
$$

Since $x_{1} \notin E$, we must have $u\left(x_{1}\right)-v\left(x_{1}\right)<u\left(x_{0}\right)-v\left(x_{0}\right)$. In particular,

$$
\varepsilon S_{\varepsilon}^{+} v\left(x_{0}\right) \geq v\left(x_{1}\right)-v\left(x_{0}\right)>u\left(x_{1}\right)-u\left(x_{0}\right)=\varepsilon S^{+} u\left(x_{0}\right),
$$

contradicting (4.4). Thus $S_{\varepsilon}^{+} u \equiv 0$ on $F$.

Since $f \leq 0$, by (4.3) we must have $S_{\varepsilon}^{-} u \equiv 0$ on $F$. Thus $u$ is constant on $\bar{B}\left(x_{0}, \varepsilon\right)$ for all $x_{0} \in F$. By (4.4), the same is true for $v$. As $F \subseteq \Omega_{\varepsilon}$ is closed, we can choose a point $x_{0} \in \partial F \subseteq F$ to obtain a contradiction.

Proof of Theorem 2.3. We first reduce to the case that $u, v \in C\left(\Omega \cup \Gamma_{N}\right)$. It is clear from Definition 2.1 that the minimum of two supersolutions is also a supersolution. Observe that the function $\varphi$ defined in (4.2) is a smooth supersolution of (2.7), where we take $k:=\|\tilde{h}\|_{L^{\infty}\left(\Omega \cup \Gamma_{N}\right)}$. Thus $\tilde{v}:=\min \{v, \varphi\}$ is a supersolution of (2.7) which is bounded above. By Lemma 4.1, we see that $\tilde{v} \in C\left(\Omega \cup \Gamma_{N}\right)$. By replacing $v$ with $\tilde{v}$, we may assume that $v \in C\left(\Omega \cup \Gamma_{N}\right)$. Similarly, we may assume that $u \in C\left(\Omega \cup \Gamma_{N}\right)$.

According to Theorem 2.2 we have

$$
a_{\varepsilon}^{-}(\beta) S_{\varepsilon}^{-} u^{\varepsilon}-a_{\varepsilon}^{+}(\beta) S_{\varepsilon}^{+} u^{\varepsilon} \leq h^{2 \varepsilon} \quad \text { and } \quad a_{\varepsilon}^{-}(\beta) S_{\varepsilon}^{-} v_{\varepsilon}-a_{\varepsilon}^{+}(\beta) S_{\varepsilon}^{+} v_{\varepsilon} \geq \tilde{h}_{2 \varepsilon} \quad \text { in } \Omega_{2 \varepsilon} \text {. }
$$

In the case $h \equiv 0 \leq \tilde{h}$, we have $h^{2 \varepsilon} \equiv 0 \leq \tilde{h}_{2 \varepsilon}$, and so we may apply Lemma 4.2 to deduce that

$$
\max _{\bar{\Omega}_{\varepsilon}}\left(u^{\varepsilon}-v_{\varepsilon}\right)=\max _{\bar{\Omega}_{\varepsilon} \backslash \Omega_{2 \varepsilon}}\left(u^{\varepsilon}-v_{\varepsilon}\right)
$$


In the case $h<\tilde{h}$, for each $r>0$ we may choose $0<\varepsilon<r / 2$ such that $h^{2 \varepsilon}<\tilde{h}_{2 \varepsilon}$ in $\Omega_{r}$ and then apply Lemma 4.2 to obtain

$$
\max _{\bar{\Omega}_{r-\varepsilon}}\left(u^{\varepsilon}-v_{\varepsilon}\right)=\max _{\bar{\Omega}_{r-\varepsilon} \backslash \Omega_{r}}\left(u^{\varepsilon}-v_{\varepsilon}\right) .
$$

Sending $\varepsilon \rightarrow 0$ in (4.5), and $\varepsilon \rightarrow 0$ followed by $r \rightarrow 0$ in (4.6), and using the upper semicontinuity of $u$ and $-v$ up to the boundary, we obtain (2.8) in the cases $h<\tilde{h}$ and $h \equiv 0$.

We are left to consider the case $\tilde{h}>0$. Define $w:=(1+\delta) v$ for $\delta>0$, and observe that $w$ satisfies

$$
\begin{cases}-\Delta_{\infty} w-\beta|D w| \geq(1+\delta) \tilde{h} & \text { in } \Omega \\ D_{\nu} w=0 & \text { on } \Gamma_{N} .\end{cases}
$$

Since $(1+\delta) \tilde{h}>h$, our argument above implies that

$$
\max _{\bar{\Omega}}(u-(1+\delta) v)=\max _{\Gamma_{D}}(u-(1+\delta) v) .
$$

Sending $\delta \rightarrow 0$, we obtain (2.8) in the case $\tilde{h}>0$. Our proof is complete.

Proof of Theorem 2.4. Define $u: \bar{\Omega} \rightarrow \mathbb{R}$ by

$u(x):=\sup \left\{w(x): w \in \operatorname{USC}(\bar{\Omega})\right.$ is a subsolution of (1.2) and $w \leq g$ on $\left.\Gamma_{D}\right\}$.

To see that $u$ is well-defined, denote $d:=\operatorname{diam}(\Omega), k:=\|f\|_{L^{\infty}\left(\Omega \cup \Gamma_{N}\right)}$, and set

$$
\gamma(t):= \begin{cases}-\frac{1}{\beta} k t+\frac{1}{\beta^{2}} k e^{-\beta d}\left(e^{\beta t}-1\right) & \text { if } \beta \neq 0, \\ \frac{1}{2} k t^{2}-d k t & \text { if } \beta=0 .\end{cases}
$$

Observe that $\gamma(0)=0, \gamma^{\prime}<0$ on $(0, d)$, and

$$
-\gamma^{\prime \prime}-\beta\left|\gamma^{\prime}\right|=-k \quad \text { in }(0, d) \text {. }
$$

Select $x_{0} \in \Gamma_{D}$ and define $\varphi(x):=\min _{\Gamma_{D}} g+\gamma\left(\left|x-x_{0}\right|\right)$. It is clear that $\varphi$ is a smooth subsolution of (1.2) and $\varphi \leq g$ on $\Gamma_{D}$. Thus $u \geq \varphi$, and in particular $u$ is bounded below. According to Lemma 4.1 $u$ is also bounded above. By construction, $u \in$ $\operatorname{USC}(\bar{\Omega})$.

We now proceed to show that $u=g$ on $\Gamma_{D}$. By construction, $u \leq g$ on $\Gamma_{D}$. To get the other inequality, select $y \in \Gamma_{D}$, and $\varepsilon>0$. For $r>0$ small enough, $g \geq g(y)-\varepsilon$ on $\Gamma_{D} \cap \bar{B}(y, r)$. With $\gamma$ as above, define

$$
\varphi^{y}(x):=g(y)-\varepsilon+\max \left\{1, \frac{\min _{\Gamma_{D}} g-g(y)}{\gamma(r)}\right\} \gamma(|x-y|) .
$$

Observe that $\varphi^{y}$ is a smooth subsolution of (1.2) and $\varphi^{y} \leq g$ on $\Gamma_{D}$. It follows that $u(y) \geq \varphi^{y}(y)=g(y)-\varepsilon$. Since $y \in \Gamma_{D}$ and $\varepsilon>0$ were arbitrary, it follows that $u \geq g$ on $\Gamma_{D}$. Moreover, since $u \geq \varphi^{y}$ and $u \in \operatorname{USC}(\bar{\Omega})$, we see that $u$ is continuous at every point $y \in \Gamma_{D}$.

We now argue that $u$ is a subsolution of the system

$$
\begin{cases}-\Delta_{\infty} u-\beta|D u|=f & \text { in } \Omega, \\ D_{\nu} u=0 & \text { on } \Gamma_{N} .\end{cases}
$$

Select a test function $\varphi \in C^{2}(\bar{\Omega})$ and a point $x_{0} \in \Omega \cup \Gamma_{N}$ such that the map $x \mapsto(u-\varphi)(x)$ has a strict local maximum at $x=x_{0}$. For sufficiently small $r>0$,

$$
\delta(r):=(u-\varphi)\left(x_{0}\right)-\max _{\bar{B}\left(x_{0}, r\right) \cap \bar{\Omega}}(u-\varphi)>0 .
$$


We also take $r>0$ to be small enough that $B\left(x_{0}, r\right) \cap \Gamma_{D}=\emptyset$. We may select a subsolution $w \leq u$ of the system (4.7) for which

$$
u\left(x_{0}\right)-w\left(x_{0}\right) \leq \frac{1}{2} \delta(r) .
$$

It follows that for small enough $r>0$, the map $x \mapsto(w-\varphi)(x)$ has a local maximum at some point $y_{r} \in B\left(x_{0}, r\right)$. We deduce that

$$
-\Delta_{\infty}^{+} \varphi\left(y_{r}\right)-\beta\left|D \varphi\left(y_{r}\right)\right| \leq f\left(y_{r}\right)
$$

Or

$$
y_{r} \in \Gamma_{N} \quad \text { and } \quad D_{\nu} \varphi\left(y_{r}\right) \leq 0 .
$$

If $x_{0} \in \Omega$, then for all small enough $r>0$ we must have (4.8). In this case we may pass to the limit $r \rightarrow 0$ and use the upper semicontinuity of the map $x \mapsto \Delta_{\infty}^{+} \varphi(x)$ to deduce that

$$
-\Delta_{\infty}^{+} \varphi\left(x_{0}\right)-\beta\left|D \varphi\left(x_{0}\right)\right| \leq f\left(x_{0}\right) .
$$

In the case that $x_{0} \in \Gamma_{N}$, then at least one of (4.8) or (4.9) must occur for infinitely many $r>0$ along any sequence $r_{j} \rightarrow 0$. In this case, we may pass to limits along a subsequence to get

$$
-\Delta_{\infty}^{+} \varphi\left(x_{0}\right)-\beta\left|D \varphi\left(x_{0}\right)\right| \leq f\left(x_{0}\right) \quad \text { or } \quad D_{\nu} \varphi\left(x_{0}\right) \leq 0 .
$$

We have verified that $u$ is a subsolution of (4.7). Since $u$ is bounded below, Lemma 4.1 implies that $u \in C\left(\Omega \cup \Gamma_{N}\right)$. Above we argued that $u$ is continuous at every point on $\Gamma_{D}$, and thus we conclude that $u \in C(\bar{\Omega})$.

We have left to show that $u$ is a supersolution of (4.7). Suppose that the map $x \mapsto(u-\varphi)(x)$ has a strict local minimum at a point $x_{0} \in \Omega \cup \Gamma_{N}$ for some test function $\varphi \in C^{2}(\bar{\Omega})$. Arguing indirectly, we suppose in addition that

$$
-\Delta_{\infty}^{-} \varphi\left(x_{0}\right)-\beta\left|D \varphi\left(x_{0}\right)\right|<f\left(x_{0}\right),
$$

and that in the case $x_{0} \in \Gamma_{N}$ we also have $D_{\nu} \varphi\left(x_{0}\right)<0$. Since $\Delta_{\infty}^{-}$is lower semicontinuous, there exists $\delta_{1}>0$ such that

$$
-\Delta_{\infty}^{-} \varphi-\beta|D \varphi|<f \quad \text { in } B\left(x_{0}, \delta_{1}\right) \cap \bar{\Omega},
$$

and $B\left(x_{0}, \delta_{1}\right) \cap \Gamma_{D}=\emptyset$. In the case $x_{0} \in \Gamma_{N}$, we may also suppose that $\delta_{1}>0$ is so small that

$$
D_{\nu} \varphi<0 \quad \text { in } B\left(x_{0}, \delta_{1}\right) \cap \Gamma_{N} .
$$

In fact, we may choose a small $c>0$ such that

$$
\begin{cases}-\Delta_{\infty}^{-} \tilde{\varphi}-\beta|D \tilde{\varphi}|<f & \text { in } B\left(x_{0}, \delta_{1} / 2\right) \cap \bar{\Omega}, \\ D_{\nu} \tilde{\varphi}<0 & \text { on } B\left(x_{0}, \delta_{1} / 2\right) \cap \Gamma_{N}, \text { if } x_{0} \in \Gamma_{N},\end{cases}
$$

where $\tilde{\varphi}(x):=\varphi(x)-\varphi\left(x_{0}\right)+u\left(x_{0}\right)+c\left(\delta_{1}^{2} / 16-\left|x-x_{0}\right|^{2}\right)$. Now define

$$
\tilde{u}(x):= \begin{cases}\max \{u(x), \tilde{\varphi}(x)\} & \text { if } x \in B\left(x_{0}, \delta_{1} / 2\right), \\ u(x) & \text { otherwise. }\end{cases}
$$

Since $\tilde{u}$ is the maximum of two subsolutions of (4.7) in the domain $B\left(x_{0}, \delta_{1} / 2\right) \cap \bar{\Omega}$ and is equal to $u$ outside of $B\left(x_{0}, \delta_{1} / 4\right) \cap \bar{\Omega}$, we see that $\tilde{u}$ is a subsolution of (4.7). Since $\tilde{u}\left(x_{0}\right)>u\left(x_{0}\right)$, we derive a contradiction to the definition of $u$. The proof that $u$ is a supersolution is complete. 
We have constructed a maximal solution $\bar{u}=u \in C(\bar{\Omega})$ to our boundary-value problem (1.2). To obtain the existence of a minimal solution $\underline{u}$, we let $w$ be the maximal solution of the problem

$$
\begin{cases}-\Delta_{\infty} w+\beta|D w|=-f & \text { in } \Omega \\ D_{\nu} w=0 & \text { on } \Gamma_{N} \\ w=-g & \text { on } \Gamma_{D}\end{cases}
$$

and set $\underline{u}=-w$. This completes the proof of Theorem 2.4.

Remark 4.3. Notice that Theorems 2.3 and 2.4 imply the uniqueness of solutions of (1.2) for generic $f$. That is, if $g \in C\left(\Gamma_{D}\right)$ and $\left\{f_{\alpha}\right\}_{\alpha \in \mathbb{R}}$ is a family of functions belonging to $C\left(\Omega \cup \Gamma_{N}\right) \cap L^{\infty}\left(\Omega \cup \Gamma_{N}\right)$ with the property that $f_{\alpha}>f_{\beta}$ whenever $\alpha>\beta$, then there exists an at-most countable set $\Lambda \subseteq \mathbb{R}$ such that the boundaryvalue problem

$$
\begin{cases}-\Delta_{\infty} u-\beta|D w|=f_{\alpha} & \text { in } \Omega \\ D_{\nu} u=0 & \text { on } \Gamma_{N} \\ u=g & \text { on } \Gamma_{D}\end{cases}
$$

possesses a unique solution for every $\alpha \in \mathbb{R} \backslash \Lambda$. For details, we refer to the proof of [2, Theorem 2.16]. See [10, Section 5] for a counterexample to uniqueness in the simple case that $\beta=0, \Gamma_{N}=\emptyset$, and $g \equiv 0$. In particular, we remark that the failure of uniqueness in general is not due to the presence of mixed boundary conditions.

Proof of Theorem 2.5. Select a test function $\varphi \in C^{2}(\bar{\Omega})$ and a point $x_{0} \in \Omega \cup \Gamma_{N}$ such that the map $x \mapsto(u-\varphi)(x)$ has a strict local maximum at $x_{0}$. We must show that

$$
-\Delta_{\infty}^{+} \varphi\left(x_{0}\right)-\beta\left|D \varphi\left(x_{0}\right)\right| \leq f\left(x_{0}\right)
$$

or

$$
x_{0} \in \Gamma_{N} \quad \text { and } \quad D_{\nu} \varphi\left(x_{0}\right) \leq 0 .
$$

We proceed by showing that (4.10) holds, under the assumption that if $x_{0} \in \Gamma_{N}$, then (4.11) fails. That is, in the case $x_{0} \in \Gamma_{N}$ we assume that $D_{\nu} \varphi\left(x_{0}\right)>0$. We may select $r>0$ so small that either $\bar{B}\left(x_{0}, r\right) \cap \partial \Omega=\emptyset$ or $\bar{B}\left(x_{0}, r\right) \subseteq \Omega \cup \Gamma_{N}$ and

$$
D_{\nu} \varphi \geq 0 \text { on } \bar{B}\left(x_{0}, r\right) \cap \Gamma_{N} .
$$

By shrinking $r>0$ further, if necessary, we may also assume that

$$
(u-\varphi)\left(x_{0}\right)=\max _{\bar{B}\left(x_{0}, r / 2\right) \cap \bar{\Omega}}(u-\varphi)>\max _{\partial B\left(x_{0}, r / 2\right) \cap \bar{\Omega}}(u-\varphi) .
$$

Notice that $\varphi$ is a solution of the system

$$
\begin{cases}-\Delta_{\infty} \varphi-\beta|D \varphi| \geq h_{j}:=-\Delta_{\infty}^{+} \varphi-\beta_{j}|D \varphi| & \text { in } B\left(x_{0}, r\right) \cap \Omega, \\ D_{\nu} \varphi \geq 0 & \text { on } \bar{B}\left(x_{0}, r\right) \cap \Gamma_{N},\end{cases}
$$

and observe that the function $h_{j} \in \operatorname{LSC}\left(\bar{B}\left(x_{0}, r\right) \cap \bar{\Omega}\right)$.

By Theorem 2.2, for any $0<\varepsilon<r / 4$ we have

$$
a_{\varepsilon}^{-}\left(\beta_{j}\right) S_{\varepsilon}^{-} \varphi_{\varepsilon}-a_{\varepsilon}^{+}\left(\beta_{j}\right) S_{\varepsilon}^{+} \varphi_{\varepsilon} \geq\left(h_{j}\right)_{2 \varepsilon} \quad \text { in } B\left(x_{0}, r-2 \varepsilon\right) \cap \bar{\Omega} .
$$

For sufficiently large $j$, we can find a small number $0<\varepsilon_{j}<r / 3$ and a point $x_{j} \in B\left(x_{0}, r / 2\right)$ such that $\varepsilon_{j} \rightarrow 0$ and $x_{j} \rightarrow x_{0}$ as $j \rightarrow \infty$ and for which the map 
$x \mapsto\left(u_{j}^{\varepsilon_{j}}-\varphi_{\varepsilon_{j}}\right)(x)$ attains its maximum on the closed ball $\bar{B}\left(x_{0}, r / 2\right)$ at the point $x=x_{j}$. This implies that

$$
\begin{aligned}
a_{\varepsilon_{j}}^{-}\left(\beta_{j}\right) S_{\varepsilon_{j}}^{-} \varphi_{\varepsilon_{j}}\left(x_{j}\right)-a_{\varepsilon_{j}}^{+}\left(\beta_{j}\right) S_{\varepsilon_{j}}^{+} \varphi_{\varepsilon_{j}}\left(x_{j}\right) & \\
& \leq a_{\varepsilon_{j}}^{-}\left(\beta_{j}\right) S_{\varepsilon_{j}}^{-} u_{j}^{\varepsilon_{j}}\left(x_{j}\right)-a_{\varepsilon_{j}}^{+}\left(\beta_{j}\right) S_{\varepsilon_{j}}^{+} u_{j}^{\varepsilon_{j}}\left(x_{j}\right) .
\end{aligned}
$$

By Theorem 2.2, we also have

$$
a_{\varepsilon_{j}}^{-}\left(\beta_{j}\right) S_{\varepsilon_{j}}^{-} u_{j}^{\varepsilon_{j}}\left(x_{j}\right)-a_{\varepsilon_{j}}^{+}\left(\beta_{j}\right) S_{\varepsilon_{j}}^{+} u_{j}^{\varepsilon_{j}}\left(x_{j}\right) \leq f_{j}^{2 \varepsilon_{j}}\left(x_{j}\right)
$$

Combining the inequalities (4.12), (4.13), and (4.14), we obtain

$$
\left(-\Delta_{\infty}^{+} \varphi-\beta_{j}|D \varphi|\right)_{2 \varepsilon_{j}}\left(x_{j}\right)=\left(h_{j}\right)_{2 \varepsilon_{j}}\left(x_{j}\right) \leq f_{j}^{2 \varepsilon_{j}}\left(x_{j}\right) .
$$

Sending $j \rightarrow \infty$ and using the lower semicontinuity of $h_{j}$ and the upper semicontinuity of $f$, we obtain (4.10), as desired.

\section{REFERENCES}

1. S. N. Armstrong and C. K. Smart, An easy proof of Jensen's theorem on the uniqueness of infinity harmonic functions, Calc. Var. Partial Differential Equations 37 (2010), no. 3, 381-384. MR2592977

2. _ A finite difference approach to the infinity Laplace equation and tug-of-war games, Trans. Amer. Math. Soc. (in press).

3. E. N. Barron, L. C. Evans, and R. Jensen, The infinity Laplacian, Aronsson's equation and their generalizations, Trans. Amer. Math. Soc. 360 (2008), no. 1, 77-101. MR2341994

4. F. Charro, J. G. Azorero, and J. D. Rossi, A mixed problem for the infinity Laplacian via tugof-war games, Calc. Var. Partial Differential Equations 34 (2009), no. 3, 307-320. MR 2471139

5. M. G. Crandall, L. C. Evans, and R. F. Gariepy, Optimal Lipschitz extensions and the infinity Laplacian, Calc. Var. Partial Differential Equations 13 (2001), no. 2, 123-139. MR1861094 (2002h:49048)

6. E. Le Gruyer, On absolutely minimizing Lipschitz extensions and PDE $\Delta_{\infty}(u)=0$, NoDEA Nonlinear Differential Equations Appl. 14 (2007), no. 1-2, 29-55. MR2346452 (2008k:35159)

7. E. Le Gruyer and J. C. Archer, Harmonious extensions, SIAM J. Math. Anal. 29 (1998), no. 1, 279-292. MR1617186 (99d:54008)

8. G. Lu and P. Wang, Infinity Laplace equation with non-trivial right-hand-side, Electron. J. Differential Equations 77 (2010), 1-12.

9. Y. Peres, G. Pete, and S. Somersille, Biased tug-of-war, the biased infinity Laplacian, and comparison with exponential cones, Calc. Var. Partial Differential Equations 38 (2010), no. 3 , 541-564.

10. Y. Peres, O. Schramm, S. Sheffield, and D. B. Wilson, Tug-of-war and the infinity Laplacian, J. Amer. Math. Soc. 22 (2009), no. 1, 167-210. MR2449057 70803

Department of Mathematics, Louisiana State University, Baton Rouge, Louisiana

E-mail address: armstrong@math.lsu.edu

Department of Mathematics, University of California, Berkeley, California 94720

E-mail address: smart@math.berkeley.edu

Department of Mathematics, University of Texas, Austin, Texas 78712

E-mail address: steph@math.utexas.edu 